\title{
Annotating Picture Description Task Responses for Content Analysis
}

\author{
Levi King \\ Indiana University \\ leviking@indiana.edu
}

\author{
Markus Dickinson \\ Indiana University \\ md7@indiana.edu
}

\begin{abstract}
Given that all users of a language can be creative in their language usage, the overarching goal of this work is to investigate issues of variability and acceptability in written text, for both non-native speakers (NNSs) and native speakers (NSs). We control for meaning by collecting a dataset of picture description task (PDT) responses from a number of NSs and NNSs, and we define and annotate a handful of features pertaining to form and meaning, to capture the multi-dimensional ways in which responses can vary and can be acceptable. By examining the decisions made in this corpus development, we highlight the questions facing anyone working with learner language properties like variability, acceptability and native-likeness. We find reliable inter-annotator agreement, though disagreements point to difficult areas for establishing a link between form and meaning.
\end{abstract}

\section{Introduction}

The (written) data of second language learners poses many challenges, whether it is being analyzed for grammatical errors (Leacock et al., 2014), for linguistic patterns (Kyle and Crossley, 2015), for content analysis (Weigle, 2013), or for interactions with intelligent computer-assisted language learning (ICALL) systems (Amaral and Meurers, 2007). One of the core issues in doing anything with learner data is the inherent amount of variability in how linguistic forms are used to convey meaning (cf., e.g., Meurers and Dickinson, 2017). It may indeed seem like learners can use an infinite variety of forms to express a particular meaning; here we attempt to investigate how large the problem of variability in one particular testing context is for computational processing.

To investigate variability and the mappings between linguistic form and meaning, in this paper we control for meaning by collecting a dataset of picture description task (PDT) responses from a number of NSs and NNSs, and we annotate a handful of features, thereby capturing the multifaceted ways in which responses can vary and can be acceptable or unacceptable. We call this the SAILS Corpus, for Semantic Analysis of Imagebased Learner Sentences - our intended use. By examining the decisions made in this corpus development, we highlight the questions facing anyone working with learner language properties such as variability, acceptability and native-likeness.

Given the form-meaning aspect of variability, we are interested in how variable linguistic behavior is for the same content, both within and between NS and NNS groups, and the potential use of NS responses to evaluate NNS responses. There is a long-standing notion that systems processing learner data would be wise to constrain the data in some way (e.g., Heift and Schulze, 2007; Somasundaran et al., 2015), but we do not know how much constraint is needed-or whether we sacrifice the possibility of observing particular learner behavior for the sake of a constraintwithout knowing more about the ways in which variation happens (cf. Bailey and Meurers, 2008).

The corpus presented here bears some similarities to other task-based learner corpora. Meurers et al. (2011) examined German learner responses to short-answer reading comprehension questions. A target answer was produced by an expert, and annotators used this target to label the meaning of responses as correct or incorrect, along with a more detailed set of labels related to form, meaning, and task appropriateness. In our own previous work (King and Dickinson, 2016, 2013), we annotated a small set of PDT responses as correct or incorrect, with incorrect responses further labeled as errors of form or meaning. Somasundaran and Chodorow (2014) presented work on PDT re- 
sponses in which respondents used provided vocabulary words. Responses were manually annotated on a holistic four point scale, and a set of five features (relating to meaning, relevance and language use) were calculated based on statistical assumptions. Somasundaran et al. (2015) performed a nearly identical analysis with transcribed texts from a six-picture narration task, but neither of these datasets is publicly available.

Our work reverses this mapping by providing manually annotated features, which we hope will be useful for mapping to holistic scores. For example, a response may present the main content of an item correctly but add imaginary details, while another may address background information not asked about in the prompt (see section 3). The acceptability of a response is thus taken as a function of several interacting features, most of which relate the text to the known semantic content. Relating to known content is distinct from typical grammatical error correction (GEC) (Leacock et al., 2014) and from more linguistically driven work such as parsing (e.g., Cahill et al., 2014; Ragheb and Dickinson, 2014), but providing the dimensions of acceptability and elucidating how they are applied provides insight for any enterprise desiring to connect learner text with semantic content, in addition to unpacking the sources of variation and of difficulty in processing a range of learner data.

In section 2 we outline the picture description task (PDT) we use, designed with items that elicit specific types of linguistic behavior. Section 3 outlines the annotation, tackling the five-dimensional scheme; inter-anntotator agreement results are in section 4 . While agreement seems reliable, highlighting areas of disagreement showcases difficult areas for establishing a link between form and meaning (cf., e.g., Meurers and Dickinson, 2017).

\section{Picture Description Task}

\subsection{PDT Stimuli}

The PDT is built around 30 cartoon-like vector graphics, or items. The images were modified to remove any non-essential detail or background; some examples are in Table 1. To factor out the influence of previous linguistic context, images are devoid of any text or symbols, with the exceptions of two images containing numerals, two with music notes, and one with a question mark. Each image depicts an ongoing or imminent action, per- formed by a person or an animal. The images are divided evenly into canonically intransitive, transitive and ditransitive actions.

Two main versions of the PDT were used. In each version, the first half contains targeted items, where questions take the form of What is $<$ subject $>$ doing?, with the subject provided (e.g., the boy, the bird). The second half contains untargeted items, where the question is, What is happening?. Collecting both versions allows one to examine response variation with and without a subject constraint, thereby informing approaches to task design and automatic content assessment (Foster and Tavakoli, 2009; Cho et al., 2013). Roughly equal numbers of targeted and untargeted responses were collected for each item.

Each half (targeted and untargeted) is introduced with instructions, including an example item with sample responses. The instructions ask participants to focus on the main event depicted in the image and for each response to be one complete sentence. The PDT was presented as an online survey, and all participants typed their responses. Participants were instructed not to use any reference materials, but were permitted to use browser-based spell checking.

\subsection{Data Collection}

A total of approximately 16,000 responses were collected from 499 participants. Of these, 141 were NNSs, recruited from intermediate and advanced writing courses for English as a Second Language students attending Indiana University. Nearly $90 \%$ of these recruits were native speakers of Mandarin Chinese, which could have important implications for conclusions drawn from the corpus. These participants performed the task in a computer lab with the researchers present. They were native speakers of Mandarin Chinese (125), Korean (4), Burmese (3), Hindi (2), and one native speaker each of Arabic, Indonesian Bahasa, German, Gujarati, Spanish, Thai and Vietnamese.

Of the 358 NS participants, 29 were personally known by the researchers. Responses from the remaining 329 NSs were purchased via an online survey platform where participants earn credits they can redeem for gift cards and prizes. Due to length restrictions for purchased surveys, these NSs each completed only half of the task, so their data is equivalent to 164.5 full participants.

In previous similar work (King and Dickinson, 


\begin{tabular}{|l|c|c|}
\hline \\
\hline What is the woman doing? [Intrans.] & A1 & A2 \\
\hline The woman is running. & 1 & 1 \\
\hline She is wearing a red shirt. & 0 & 0 \\
\hline Trying to run from her bad decisions & 1 & 0 \\
\hline \hline
\end{tabular}

Table 1: Test sample items and example responses with Core Event annotations from Annotators 1 and 2.

2013), NSs were found to produce less variation than NNSs. Many NSs provided identical responses or ones very similar to the most canonical way of expressing the main action. One purpose of gathering the data is to be able to assess NNS response content by comparing it against the NS responses; thus, NSs were asked to provide two nonidentical responses, in the hopes that this would result in more examples of native-like responses for the NNS responses to compare against.

\begin{tabular}{|l||l|l||l|l|}
\hline \multicolumn{1}{|c||}{} & \multicolumn{2}{c||}{ Targeted } & \multicolumn{2}{c|}{ Untargeted } \\
\hline Set & NS & NNS & NS & NNS \\
\hline \hline Intrans & 0.628 & 0.381 & 0.782 & 0.492 \\
\hline Trans & 0.752 & 0.655 & 0.859 & 0.779 \\
\hline Ditrans & 0.835 & 0.817 & 0.942 & 0.936 \\
\hline
\end{tabular}

Table 2: NS and NNS type-to-token ratios (TTR) for complete responses (not words), for all the data.

To examine the degree of variation among the NS and NNS groups in the current study, typeto-token ratios (TTR) were calculated on the response level (ignoring case and final punctuation) for the entire set of items, shown in Table 2. For each data point in the table, the corpus contains roughly 150 NS responses and 70 NNS responses. To control for this imbalance and its effect on the likelihood of seeing new responses, the TTR was calculated for each item based on a random sample of 50 responses. Specifically, we randomly sampled 50 responses, calculated the TTR, and averaged them. The scores in in Table 2 show that, in all cases, the NS set shows a greater degree of response variation, meaning that asking for two responses is an effective way of collecting a broader range of NS responses.

The ratios show the direct relationship between the complexity of the event portrayed (represented here as intransitive, transitive and ditransitive) and the degree of variation elicited. In all cases, TTR increases with this complexity. Interestingly, this trend seems more pronounced in the NNS responses; in the targeted NNS responses, the TTRs for intransitive and ditransitive items are 0.381 and 0.817 , respectively, compared to 0.628 and 0.835 for NS responses. The ratios also show that in all cases, variation is greater for untargeted items than it is for targeted items. In other words, asking about a particular subject in the prompt question does constrain the variety of responses.

\section{Annotation scheme}

The data were annotated with the aim of providing information that would be useful for the automatic assessment of NNS responses via comparison with NS responses. The annotation scheme was developed through an iterative process of annotation, discussion and revision, with input from two annotators and multiple language professionals. The initial scheme was planned as a three- 
point scale, ranging from accurate and native-like (2) to accurate but not native-like (1) to not accurate (0). This proved problematic, however, as $a c$ curacy and native-likeness could not be adequately defined and applied to the data. For example, in the middle picture of Table 1, it is not clear how accurate or native-like She is happy with the dog is. Grammatically, it is native-like, but it does not seem like an appropriate answer to the question, What is the woman doing?

To address the specifics of appropriate answers, five binary features were eventually settled on, with each feature having some relation to the original concepts of accuracy and native-likeness. A set of annotation guidelines were produced with definitions, rules and examples for each feature. For most features, the rules for targeted and untargeted items vary slightly; the untargeted rules are generally less strict. The features and brief descriptions are listed here and discussed further in the following sections:

1. Core Event: Does the response capture the core event depicted in the image? Core events are not pre-defined but should be fairly obvious given the nature of the images. The response should link an appropriate subject to the event. In the top picture of Table 1, The woman is running clearly captures the core event, while She is wearing a red shirt is irrelevant to the event happening.

2. Verifiability: Does the response contain only information that is true and verifiable based on the image? Inferences should not be speculations and are allowed only when necessary and highly probable, as when describing a familial relationship between persons depicted in the image. For example, in Table 1 , She is wearing a red shirt conveys information that is irrelevant to the core event but is nonetheless recoverable from the image (annotation=1), while Trying to run from her bad decisions has information that cannot be inferred from the picture.

3. Answerhood: Does the response make a clear attempt to answer the question? This generally requires a progressive verb. For targeted items, the subject of the question, or an appropriate pronoun, must be used as the subject of the response. For example, The dog is happy is answering a question other than What is the woman doing? (Table 1).

4. Interpretability: Does the response evoke a clear mental image (even if different from the item image)? Any required verb arguments must be present and unambiguous. For example, The map is hard to read is too vague to generate a clear mental image (Table 1).

5. Grammaticality: Is the response free from errors of spelling and grammar? In our data set, this is a relatively straightforward feature to annotate (see section 4).

Example annotations In Table 3, we see example responses with all five features annotated, illustrating each feature's distinctiveness from the others. For example, for He is eating food one can generate a mental picture, e.g., of someone chewing (interpretability=1), but the pizza is important to the item image (core event $=0$ ). As another example, He may get fat eating pizza seems to be addressing a question about the consequences of the eating action (answerhood $=0$ ) and talks about hypotheticals not in the picture (verifiability $=0$ ). Teasing apart these annotations is the focus of the next section.

\section{Agreement}

Two annotators participated in the annotation. Both are native speakers of (US) English, and each has several years of language teaching experience with both children and adult learners. Annotator 1 (A1) annotated the complete corpus. Annotator 2 (A2) annotated only the development set and the test set, data subsets described next.

Three items were used as a development set for creating and revising the annotation scheme. These items were also used as examples in the guidelines. They represent one intransitive, one transitive and one ditransitive item. Both annotators annotated portions of the development set multiple times throughout the process, discussing and adjudicating disagreeing annotations before moving on to the test set, which was completed without consultation between the annotators.

The test set parallels the development set and consists of one intransitive, one transitive and one ditransitive item; it is shown in Table 1. Agreement and Cohen's kappa scores are given in Table 4 , broken down by different criteria. We will now walk through these results. 


\begin{tabular}{|c|c|c|c|c|c|}
\hline What is the boy doing? & $\mathrm{C}$ & $\mathrm{V}$ & $\overline{\mathrm{A}}$ & I & $\mathrm{G}$ \\
\hline He is eating food. & 0 & 1 & 1 & $\overline{1} 1$ & $\overline{1}$ \\
\hline eatting. & 0 & 1 & 1 & 1 & 0 \\
\hline $\begin{array}{l}\text { The child is about to eat } \\
\text { pizza. }\end{array}$ & 1 & 1 & 0 & 1 & 1 \\
\hline $\begin{array}{l}\text { He may get fat eating } \\
\text { pizza. }\end{array}$ & 1 & 0 & 0 & 1 & 1 \\
\hline What is happening? & $\overline{\mathrm{C}}$ & $\overline{\mathrm{V}}$ & $\overline{\mathrm{A}}$ & 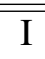 & $\overline{\mathrm{GG}}$ \\
\hline Child is eating pizza. & 1 & 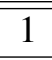 & 1 & 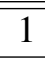 & 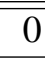 \\
\hline Tommy is eating pizza. & 1 & 0 & 1 & 1 & 1 \\
\hline $\begin{array}{l}\text { The boy's eating his fa- } \\
\text { vorite food. }\end{array}$ & 0 & 0 & 1 & 0 & 1 \\
\hline $\begin{array}{l}\text { Pizza is this boy's fa- } \\
\text { vorite food. }\end{array}$ & 0 & 0 & 0 & 0 & 1 \\
\hline
\end{tabular}

Table 3: Targeted and untargeted sample responses from the development set transitive item, shown with adjudicated annotations for the five features: core event $(C)$, verifiability $(V)$, answerhood $(A)$, interpretability $(I)$ and grammaticality $(G)$.

\subsection{Transitivity}

Comparing the intransitive, transitive and ditransitive items reveals an association between agreement and item complexity. The highest raw agreement and Cohen's kappa scores are found with the intransitive item $(97.8 \%, \kappa=0.910)$ and the lowest with the ditransitive $(92.4 \%, \kappa=0.764)$.

This is as expected, as ditransitive sentences are longer and have more verbal arguments, making for more opportunities for responses to vary (see Table 2), and thus more opportunities for annotators to disagree on a response. This trend also matches annotator feedback: both ranked the ditransitive item as the most difficult to annotate overall, and the intransitive as the easiest.

\subsection{Targeting}

Grouping the annotations into targeted and untargeted sets, the raw agreement scores are compara- ble (94.9\% vs. $95.2 \%)$. However, despite a greater degree of response variation, the untargeted group has a higher kappa score ( 0.872 vs. 0.823$)$. When asked to compare the annotations, A2 noted that targeted responses require more concentration and closer consultation of the guidelines. For example, answerhood does not allow for targeted responses to modify the subject provided in the question in any way, whereas in answering What is happening?, the respondent is free to speak of characters in the pictures in many different ways. Both A1 and A2 thus describe the annotation of untargeted items as less restrictive.

\subsection{Features}

Grouped by feature, the annotations all show raw agreement scores above $91 \%$ and Cohen's kappa scores above 0.74 (Table 4). For future use of this corpus in content assessment, these kappa scores are comfortably above the 0.67 suggested as a baseline for meaningful, reliable agreement (Landis and Koch, 1977; Artstein and Poesio, 2008). We discuss each feature in turn, highlighting difficulties in coming to an agreement, as such disagreements illustrate some sources of variability.

Core event Isolating whether the main content of the picture is being described or not, the core event feature is the most relevant of the five for content assessment. All five features are skewed toward yes annotations, but with an average yes rate of $72.5 \%$, core event is the least skewed; i.e., more responses receive a no annotation for core event than for any other feature.

Core event has the second lowest interannotator agreement kappa score, at 0.808 . This is somewhat lower than expected, as the preadjudication development set score was 0.889 . This appears to be largely attributable to the difficulty of the ditransitive item, challenging for both participants and annotators (section 4.1).

The main issue in this case has to do with the amount of specificity required to be the core event. The development set item depicts a man delivering a package to a woman, and most responses describe this as such a transaction, using give, deliver or receive. The test set item shows a man giving directions to a woman (Table 1), and this resulted in a greater degree of variation. Many (particularly NNS) responses portray this not as a canonical giving directions event but as pointing, helping a lost person or reading a map, with $\mathrm{A} 2$ more 


\begin{tabular}{|l|l|l|l|l||l|l||l|}
\hline Set & Total & A1Yes & A2Yes & AvgYes & Chance & Agree & Kappa \\
\hline \hline Intransitive & 2155 & 0.863 & 0.855 & 0.859 & 0.758 & 0.978 & 0.910 \\
\hline Transitive & 2155 & 0.780 & 0.774 & 0.777 & 0.653 & 0.949 & 0.853 \\
\hline Ditransitive & 2155 & 0.812 & 0.786 & 0.799 & 0.678 & 0.924 & 0.764 \\
\hline \hline Targeted & 3390 & 0.829 & 0.818 & 0.824 & 0.709 & 0.949 & 0.823 \\
\hline Untargeted & 3075 & 0.806 & 0.790 & 0.798 & 0.678 & 0.952 & 0.872 \\
\hline \hline Core Event & 1293 & 0.733 & 0.717 & 0.725 & 0.601 & 0.923 & 0.808 \\
\hline Verifiability & 1293 & 0.845 & 0.817 & 0.831 & 0.719 & 0.968 & 0.884 \\
\hline Answerhood & 1293 & 0.834 & 0.831 & 0.833 & 0.721 & 0.982 & 0.936 \\
\hline Interpretability & 1293 & 0.818 & 0.787 & 0.802 & 0.682 & 0.919 & 0.744 \\
\hline Grammaticality & 1293 & 0.861 & 0.872 & 0.866 & 0.768 & 0.960 & 0.827 \\
\hline
\end{tabular}

Table 4: Agreement scores broken down by different properties of the test set: total annotations (Total), yes annotations for Annotator 1 and 2 (A1Yes, A2Yes), average yes annotations (AvgYes), total expected chance agreement for yeses and nos (Chance), actual raw agreement (Agree) and Cohen's kappa (Kappa).

likely to accept these less specific descriptions.

Similarly, but to a lesser extent, the transitive item, which shows a woman hugging a dog (Table 1), resulted in disagreements where A2 accepts the word pet as the object, but A1 rejects such responses as too vague. Despite the acceptable scores for core event agreement, the fact that many disagreements hinge on particular word choice or annotators having minor differences in interpretation of the event suggest that greater agreement could be achieved by providing annotators with suggestions about the acceptable content for each response. In other words: by more clearly determining the desired level of specificity of a response-for the verb or its argumentsagreement could be higher. The desired specificity may vary in accordance with the intended use of the annotations; in the current annotations, the standard discussed between annotators and in the guidelines included pragmatic considerations like naturalness, native-likeness and effort.

Verifiability On the flipside of the question of whether the core semantic content is expressed is the question of whether any extraneous content is added, or any content used in a way which cannot be verified from the picture. The average yes rate for verifiability is $83.1 \%$, making it the third most skewed feature.

The raw agreement score is $96.8 \%$, and the kappa score is 0.884. By both measures, this is the second highest agreement score, after answerhood. Of 42 disagreements for verifiability, annotators agree that at least eight are avoidable. Of these, five involve the in- correct use of plurals. For example, A1 accepted $A$ man is pointing the way for the women, when the image shows only one woman, but the guidelines reject such responses. Two other errors stem from inaccuracy, with respondents referring to a dog in the illustration as a cat. Each annotator incorrectly accepted one such response. One disagreement involved the misspelling of a crucial object: The woman is holding the pat. It is unclear whether pet or cat was intended. This should render the response unverifiable, but A1 accepted it.

The remaining disagreements are attributable to different opinions about inferences, with A2 being, in general, more strict. For the ditransitive item, for example, both annotators accept responses that refer to the woman as a hiker, but only A1 accepts responses where the man and woman are collectively referred to as hikers. For the intransitive item depicting a woman running, A1 accepts multiple responses that refer to this as a race, as well as responses that infer the runner's motivation (fitness, leisure, etc.).

Answerhood Capturing the semantic content of the picture isn't the only criterion for determining the quality of a response; the answerhood feature was added largely as a way to identify responses that simply do not follow the instructions. Such responses tend to be: i. responses that do not directly answer the given question, perhaps by reframing the perspective so that it seems like a different question was asked; ii. responses that are gibberish or very low-effort and entered only so the participant can proceed to the next item; or iii. "troll" responses that attempt to be funny or ob- 
scene at the cost of attempting a direct answer.

The majority of participants do attempt to follow the instructions and answer the question, however, and it is unsurprising that this feature skews strongly toward yes annotations and results in the highest raw agreement $(98.2 \%)$ and kappa (0.936) scores among the five features.

Of 23 disagreements, seven stem from one annotator failing to enforce the requirement that a targeted response subject be either an appropriate pronoun or the exact subject given in the question, without adjectives, relative clauses or other modifiers. Given the question What is the woman doing?, for example, the responses The lady is running and The woman who in pink is running were incorrectly accepted by one annotator. While this criterion may seem strict, this subject-identity rule separates the task of identifying an attempt to answer the question from the task of verifying information (see verifiability above).

Another ten disagreements involve responses lacking a progressive verb, generally required as an indication that the response refers to the specific action in the image and does not merely describe a state or a general truth (cf., e.g., The woman is running vs. The woman runs). Annotator fatigue thus accounts for the majority of answerhood disagreements.

Interpretability The average yes rate for interpretability is 0.802 ; only core event is less skewed: responses were thus also more likely to be unacceptable. The raw agreement score is $91.9 \%$ and kappa is 0.744 , the lowest scores among the five features. This was anticipated, because interpretability is perhaps the most difficult to define, leaving room for annotators' personal judgments. Annotators must decide whether a given response evokes a clear mental image, regardless of how well that mental image matches the PDT image. In this way, responses such as The man is working which may be completely verifiable may still fall short, in that the man could be picking fruit, building a bridge, and so forth.

The guidelines place some restrictions on what it means to be a clear mental image. To begin with, if one were to illustrate the response, the result would be a complete, representational, canonical image. It would not be necessary to guess at major elements, like subjects or objects. All necessary semantic arguments would be identifiable from the sentence and thus not obscured or out of the frame in the mental image. Vague language should be avoided, but human gender does not need to be specified, especially when a non-gendered word like doctor or teacher is natural.

Consider a response like $A$ woman is receiving a package. By these criteria, the response is annotated as 0 because the person or entity delivering the package is not specified, and an illustrator would need to either guess or compose the image with the deliverer oddly out of the frame. A man is delivering a package, on the other hand, would be accepted. An illustrator could simply show a delivery person carrying a package, as an indirect object would not be necessary for the verb deliver.

Among the 105 annotator disagreements, fatigue accounts for roughly 30 ; this is difficult to determine precisely because annotators expressed difficulty in identifying a single root cause for many disagreements. Those that are clearly attributable to annotator error tend to involve responses with some internal inconsistency, as with subject-verb disagreements, where the number of the subject is uninterpretable. Among true disagreements, the level of specificity is often the point of contention, as with core event. For example, A1 accepted several transitive item responses with the verb love, as in The woman loves her dog (Table 1). A2 explained that these are too vague to illustrate as an action; A1 disagreed, and this seems to indicate differing judgments regarding the use of love as a dynamic verb.

Grammaticality The grammaticality feature is the most heavily skewed one, with an average yes rate of $86.6 \%$. As the only non-semantic annotation, this is perhaps not surprising.

Grammaticality has a raw agreement score of 96.0\% and a kappa of 0.827. Among 52 disagreements, annotators concurred in discussion that 19 involve an avoidable annotator error. These are primarily responses with typos, misspellings, subject-verb disagreement and bare nouns, all rejected by the annotation rules. Such cases are likely attributable to annotator fatigue.

The remainder reflect an unavoidable level of disagreement. Many of these stem from differing interpretations of bare nouns as either errors or as acceptable mass nouns, as in The man is giving direction to the tourist. In several cases, annotators disagree over prepositions, which are known to be a common source of disagreement and pose spe- 
cial challenges in the context of learner language (Tetreault and Chodorow, 2008a,b). For example, annotators could not agree on the grammaticality of the prepositions in The girl is asking for help to the man and The girl is hugging with her cat.

\subsection{NS \& NNS responses}

Agreement scores were also calculated separately for NS and NNS responses, as shown in Table 5. Comparing the average rate of yes annotations shows that the NNSs outperform the NSs by between roughly $8 \%$ and $12 \%$ on all features except grammaticality. It is not surprising that NSs outperform NNSs on this feature $(90.2 \%$ to $79.3 \%$ ), but to account for their superior performance on the other features, one must consider the fact that the NNSs were recruited from ESL courses and performed the task with peers and researchers present. The NNSs were more likely to make a good faith effort than the NSs, the majority of whom performed the task anonymously and remotely. Furthermore, with twice as many responses to provide for each item for NSs, fatigue and boredom may have been a contributing factor.

\begin{tabular}{|l||l|l||l|l|}
\hline \multicolumn{1}{|l||}{} & \multicolumn{2}{c||}{ AvgYes } & \multicolumn{2}{c|}{ Kappa } \\
\hline Set & NS & NNS & NS & NNS \\
\hline \hline Core & 0.686 & 0.805 & 0.819 & 0.767 \\
\hline Verif & 0.807 & 0.882 & 0.904 & 0.819 \\
\hline Answer & 0.800 & 0.899 & 0.928 & 0.961 \\
\hline Interp & 0.764 & 0.881 & 0.752 & 0.697 \\
\hline Gramm & 0.902 & 0.793 & 0.786 & 0.863 \\
\hline
\end{tabular}

Table 5: NS and NNS test set responses: average yes annotations (AvgYes) and Cohen's kappa (Kappa).

Raw agreement scores are high among both groups, ranging from $91 \%$ to $99.3 \%$ (not shown). Notably, for core event, verifiability and interpretability, kappa scores are higher for NS responses than for NNS ones; i.e., annotators agree more on NS responses for these features. It may be no coincidence that these three features are the most closely tied to meaning, while answerhood gets at pragmatics and grammaticality focuses on form correctness.

The lower kappa score for NS answerhood is also attributable to task effects, as a second response (as required of NSs) is more likely to be off topic or in bad faith. For grammaticality, kappas for annotator agreement are higher for NNS responses. A relatively low rate of expected (chance) agreement contributes to this fact. Additionally, annotators note that many grammar problems with NNS responses are obvious (e.g., The man who in yellow is showing the way to a girl, see Table 1), but the few grammar problems in NS data are mostly typos and more easily overlooked due to fatigue (e.g., The man is giving ditections).

\section{Conclusion}

The SAILS corpus presented here was developed with specific research in mind, but also in the hopes that it may be used to address a broad range of questions. We have demonstrated here a set of binary features that were successfully implemented with reliable levels of inter-annotator agreement. These features were defined with an eye toward content analysis and ICALL, but we believe the annotations and raw responses could find uses in question answering, dialogs, pragmatic modeling, visual references and other challenges in natural language processing. The feature set could also be expanded to better suit other purposes, and the task could easily be extended to include new items. Guidelines, task materials and annotation tools are included with the corpus. ${ }^{1}$

A number of lessons have been learned in this process, and as we intend this work to be extendable, a few suggestions are in order. The inclusion of any symbols or numerals should be avoided as they resulted in response complications; some participants gave clever "meta" responses (She's breathing in music notes, rather than She's singing), and others focused on the symbols rather than the abstract concepts they represent (The teacher is teaching ' $2+2=4$ ', rather than The teacher is teaching math). The comparison of crowdsourced NS data with the data of known NS participants and the NNS student data makes it clear that motivations and task environment can affect the quality of responses.

Additionally, more clearly defining acceptable core events could lessen the ambiguity for annotators. While we intend the NS responses collected here to be useful for comparing with NNS responses and addressing related research questions, for specific applications like language testing, the use of expert annotators and constructed reference materials or gold standards may be more appropriate (Somasundaran and Chodorow, 2014).

\footnotetext{
${ }^{1}$ https://github.com/sailscorpus/sails
} 


\section{References}

Luiz Amaral and Detmar Meurers. 2007. Conceptualizing student models for ICALL. In Proceedings of the 11th International Conference on User Modeling, Corfu, Greece.

Ron Artstein and Massimo Poesio. 2008. Survey article: Inter-coder agreement for computational linguistics. Computational Linguistics, 34(4).

Stacey Bailey and Detmar Meurers. 2008. Diagnosing meaning errors in short answers to reading comprehension questions. In The 3rd Workshop on Innovative Use of NLP for Building Educational Applications (ACLO8-NLP-Education), pages 107-115, Columbus, $\mathrm{OH}$.

Aoife Cahill, Binod Gyawali, and James Bruno. 2014. Self-training for parsing learner text. In Proceedings of the First Joint Workshop on Statistical Parsing of Morphologically Rich Languages and Syntactic Analysis of Non-Canonical Languages, pages 6673, Dublin, Ireland. Dublin City University.

Yeonsuk Cho, Frank Rijmen, and Jakub Novák. 2013. Investigating the effects of prompt characteristics on the comparability of toefl ibtl integrated writing tasks. Language Testing, 30(4):513-534.

Pauline Foster and Parvaneh Tavakoli. 2009. Native speakers and task performance: Comparing effects on complexity, fluency, and lexical diversity. Language learning, 59(4):866-896.

Trude Heift and Mathias Schulze. 2007. Errors and Intelligence in Computer-Assisted Language Learning: Parsers and Pedagogues. Routledge.

Levi King and Markus Dickinson. 2013. Shallow semantic analysis of interactive learner sentences. In Proceedings of the Eighth Workshop on Innovative Use of NLP for Building Educational Applications, pages 11-21, Atlanta, Georgia.

Levi King and Markus Dickinson. 2016. Shallow semantic reasoning from an incomplete gold standard for learner language. In Proceedings of the 11th Workshop on Innovative Use of NLP for Building Educational Applications, pages 112-121.

Kristopher Kyle and Scott A Crossley. 2015. Automatically assessing lexical sophistication: Indices, tools, findings, and application. Tesol Quarterly, 49(4):757-786.

J Richard Landis and Gary G Koch. 1977. The measurement of observer agreement for categorical data. biometrics, pages 159-174.

Claudia Leacock, Martin Chodorow, Michael Gamon, and Joel Tetreault. 2014. Automated Grammatical Error Detection for Language Learners, second edition. Synthesis Lectures on Human Language Technologies. Morgan \& Claypool Publishers.
Detmar Meurers and Markus Dickinson. 2017. Evidence and interpretation in language learning research: Opportunities for collaboration with computational linguistics. Language Learning. Special Issue on Language learning research at the intersection of experimental, corpus-based and computational methods, 67(S1):66-95.

Detmar Meurers, Ramon Ziai, Niels Ott, and Janina Kopp. 2011. Evaluating answers to reading comprehension questions in context: Results for german and the role of information structure. In Proceedings of the TextInfer 2011 Workshop on Textual Entailment, pages 1-9. Association for Computational Linguistics.

Marwa Ragheb and Markus Dickinson. 2014. The effect of annotation scheme decisions on parsing learner data. In Proceedings of the 13th International Workshop on Treebanks and Linguistic Theories (TLT13), Tübingen, Germany.

Swapna Somasundaran and Martin Chodorow. 2014. Automated measures of specific vocabulary knowledge from constructed responses ('Use these words to write a sentence based on this picture'). In Proceedings of the Ninth Workshop on Innovative Use of NLP for Building Educational Applications, pages 1-11, Baltimore, Maryland.

Swapna Somasundaran, Chong Min Lee, Martin Chodorow, and Xinhao Wang. 2015. Automated scoring of picture-based story narration. In Proceedings of the Tenth Workshop on Innovative Use of NLP for Building Educational Applications, pages 42-48, Denver, Colorado. Association for Computational Linguistics.

Joel Tetreault and Martin Chodorow. 2008a. Native judgments of non-native usage: Experiments in preposition error detection. In Coling 2008: Proceedings of the workshop on Human Judgements in Computational Linguistics, pages 24-32, Manchester, UK. Coling 2008 Organizing Committee.

Joel Tetreault and Martin Chodorow. 2008b. The ups and downs of preposition error detection in ESL writing. In Proceedings of COLING-08, Manchester.

Sara Cushing Weigle. 2013. English language learners and automated scoring of essays: Critical considerations. Assessing Writing, 18(1):85-99. 